Supporting Information

\title{
Mechanical Effect on Gene Transfection Based on Dielectric Elastomer Actuator
}

Chao Gao, ${ }^{\dagger \#}$ Zhichao Li, ${ }^{\dagger \#}$ Jiang Zou, ${ }^{\not \#}$ Jin Cheng, ${ }^{\dagger}$ Kai Jiang, ${ }^{\dagger}$ Changrun Liu, ${ }^{\dagger}$ Guoying Gu, ${ }^{\ddagger *}$ Wei Tao, ${ }^{\dagger *}$ Jie Song ${ }^{\dagger} \xi *$

'Institute of Nano Biomedicine and Engineering, Department of Instrument Science and Engineering, School of Electronic Information and Electrical Engineering, Shanghai Jiao Tong University, Shanghai 200240, People's Republic of China

${ }^{\ddagger}$ Robotics Institute, School of Mechanical Engineering, Shanghai Jiao Tong University, Shanghai, 200240, People's Republic of China

${ }^{\S}$ Institute of Cancer and Basic Medicine (ICBM), Chinese Academy of Sciences; The Cancer Hospital of the University of Chinese Academy of Sciences, Hangzhou, Zhejiang 310022, People's Republic of China

${ }^{\#}$ C.G., Z. L. and J. Z. contributed equally

*Corresponding Authors: guguoying@sjtu.edu.cn, taowei@sjtu.edu.cn and sjie@sjtu.edu.cn. 
(a)

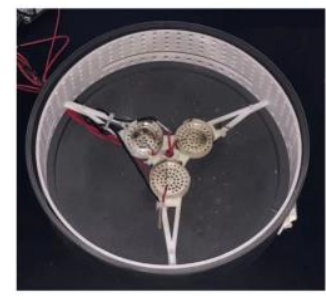

(b)

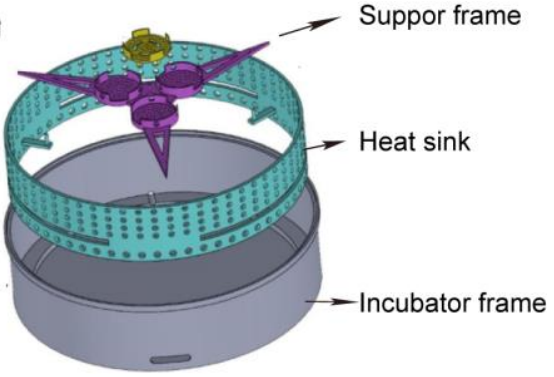

Figure S1. High-throughput cell incubator. (a) Picture of the incubator. (b) Exploded view of the incubator assembly.

\section{Temperature distribution in the culture device}

Using the infrared thermometer, we measured the temperature distribution in the culture device, and the temperature fluctuation within two hours was counted. The controller temperature was set to $32{ }^{\circ} \mathrm{C}$

(a)

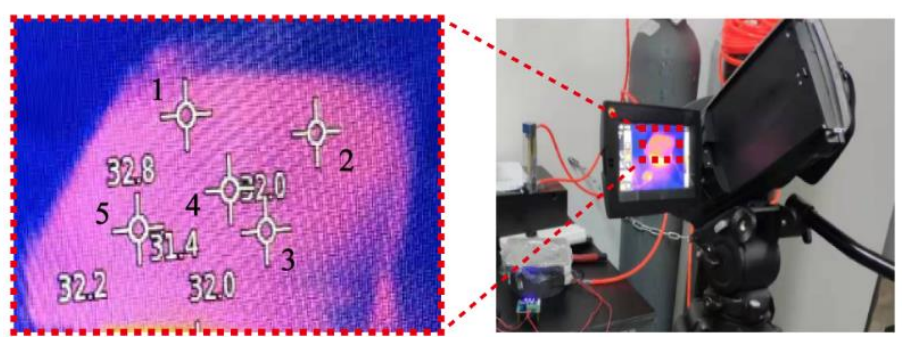

(b)

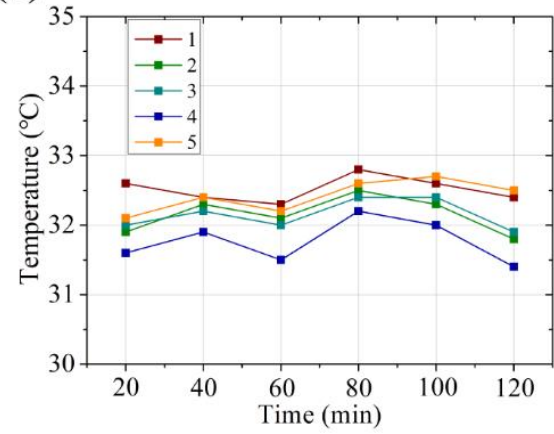

(c)

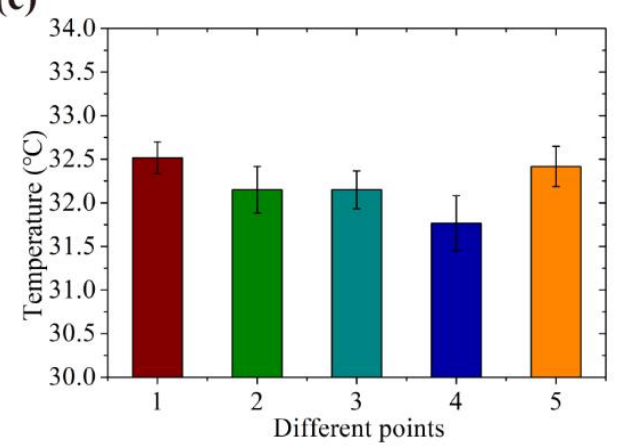

Figure S2. The temperature distribution in the cell culture device. (a) Five points in different areas around the culture dish were selected for temperature measurement. (b) Temperature distribution at five points within two hours. (c) The mean temperature and fluctuation of each point. 


\section{The deformation distribution of the membrane}

(a)

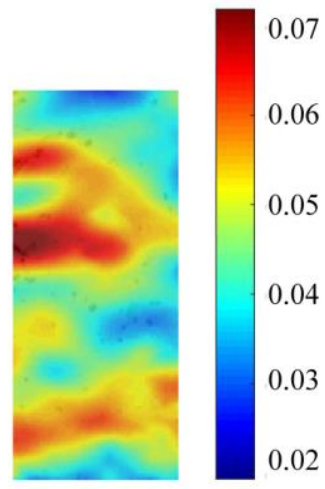

(b)

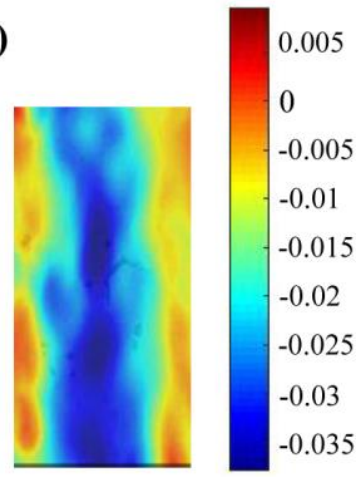

Figure S3. Stretching and compressing deformation of the membrane. (a) The deformation distribution on the stretching region. (b) The deformation distribution on the compressing region. The measurement was made at $140 \mathrm{~V} / \mu \mathrm{m}$.

\section{Cell viability}

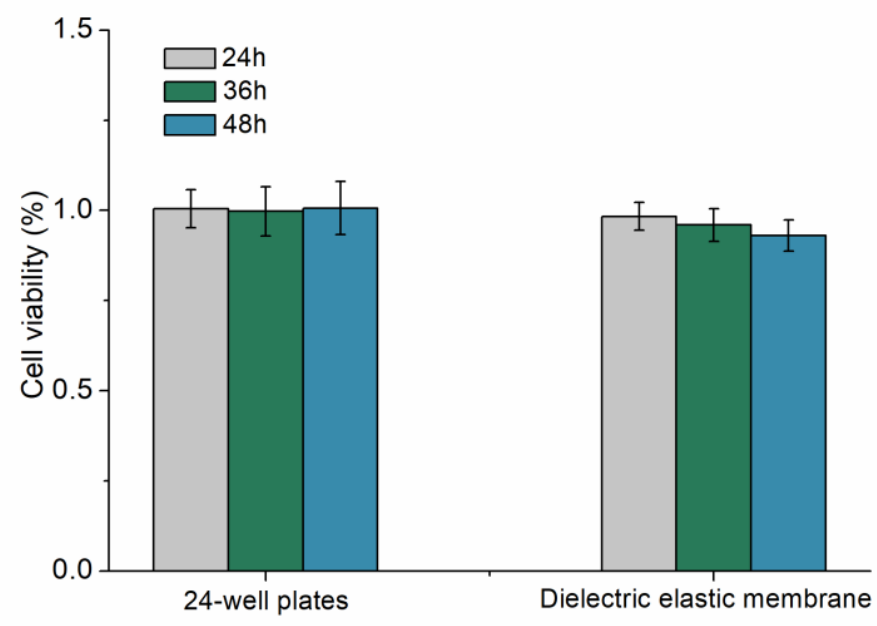

Figure S4. Cell viability in DEA based bioreactors. Cells were incubated in standard 24-well plates and dielectric elastomer membrane, and the cell viability were compared after 24, 36 and 48 hours. 


\section{Deformation of cells}

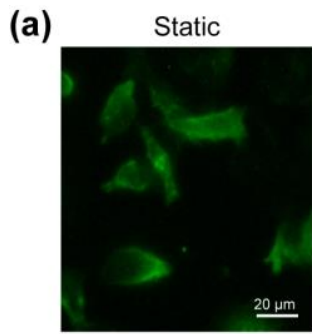

(b)

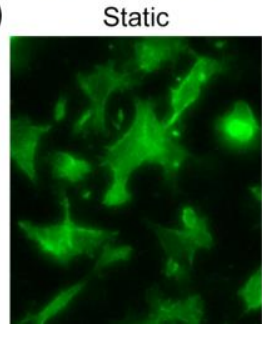

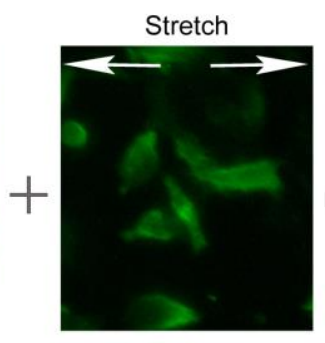

Compress

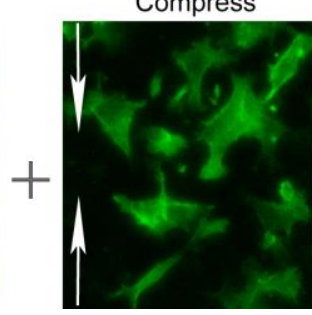

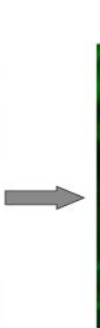

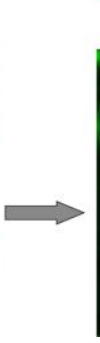

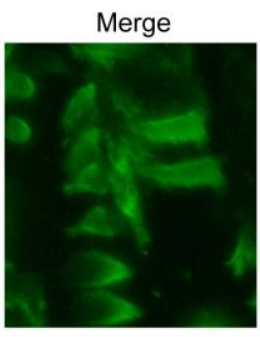

Merge

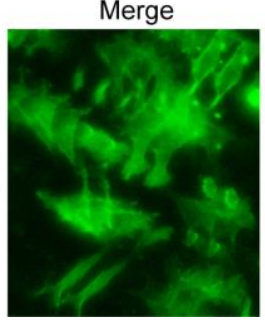

Minus coincident area

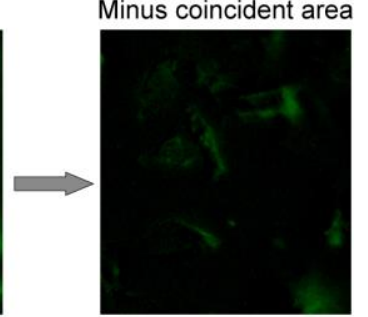

Minus coincident area

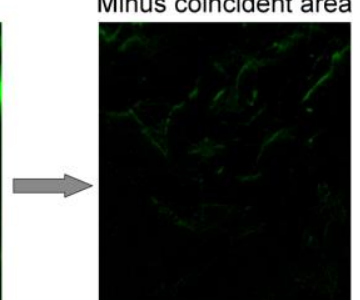

Figure S5. Deformation diagrams of stimulated cytoskeletons. (a) The cytoskeletons were stretched as the deformation of dielectric elastomer membrane. (b) The cytoskeletons were compressed as the compression of dielectric elastomer membrane. The electric field was $140 \mathrm{~V} / \mu \mathrm{m}$. 


\section{Effect of fringing electric field on EGFP transfection efficiency of A549 cells}

We tested two different electric field intensities, $100 \mathrm{~V} / \mu \mathrm{m}$ and $160 \mathrm{~V} / \mu \mathrm{m}$, respectively. The loading frequency is $10 \mathrm{mHz}$ with a $100 \%$ duty cycle. The plasmid and liposomes were added quantitatively and the operation process of the two groups was exactly the same.

(a)

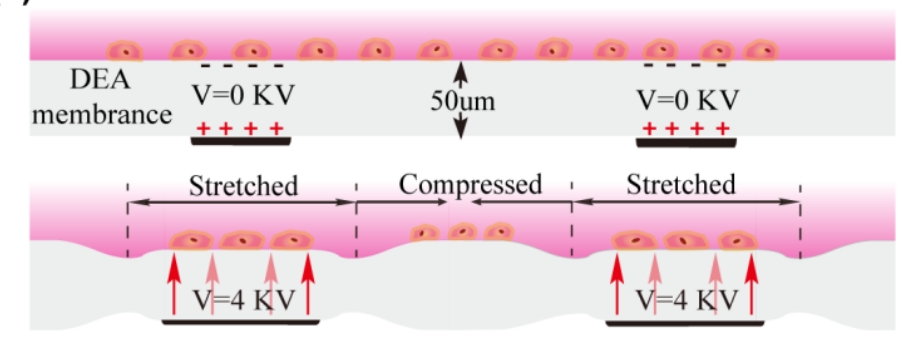

(b)

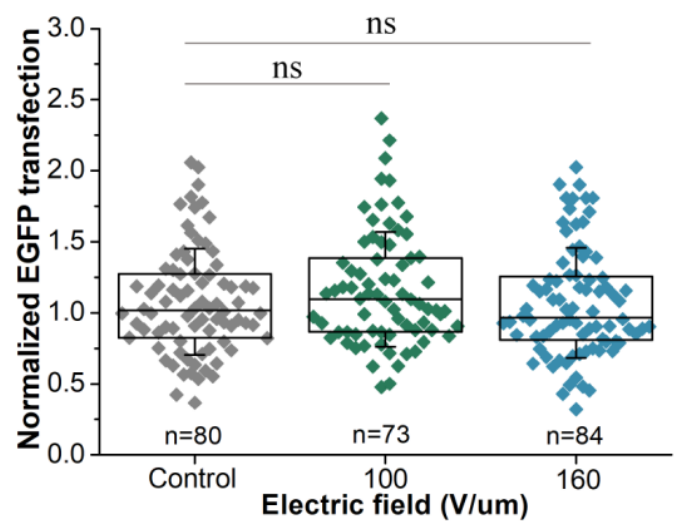

Figure S6. Effect of fringing electric field on EGFP transfection efficiency. (a) The mechanism of dielectric elastomer actuator. (b) Transfection results under two different electric field intensity were counted after 24 hours. Two different electric field, $100 \mathrm{~V} / \mu \mathrm{m}$ and $160 \mathrm{~V} / \mu \mathrm{m}$ were selected at a $10 \mathrm{mHz}$ frequency. 


\section{Endocytosis inhibition assay}

Firstly, the cells were pretreated with chlorpromazine, genistein and wortmannin for 0.5 hour at $37^{\circ} \mathrm{C}$, then the transfection reagent was added and incubated for 1 hour. The inhibitory effect of each pathway was analyzed 24 hours later for EGFP expression.

(a)

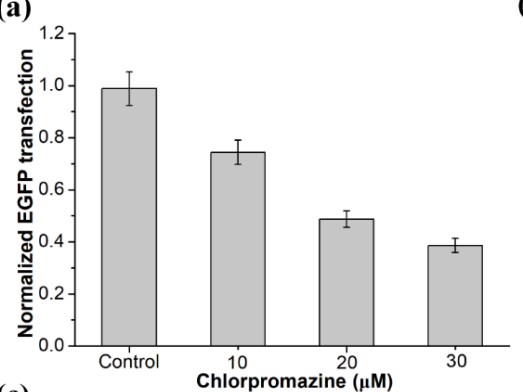

(c) (b)

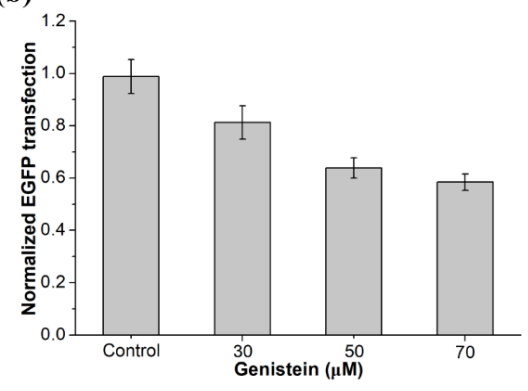

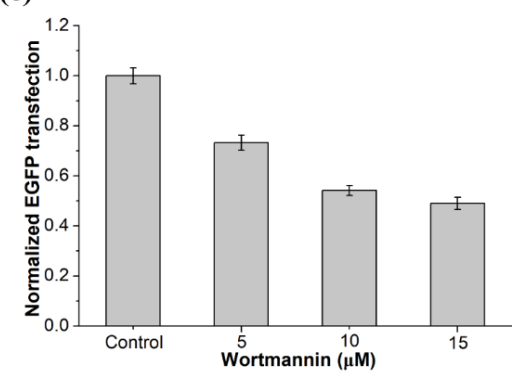

Figure S7. The effect of pathway inhibition on EGFP transfection efficiency. (a)-(c) represents the inhibitory effect on clathrin-, caveolae-, and macropinocytosismediated endocytosis pathways, respectively. 